\title{
Overexpression of FGFR3 in HPV-positive Tonsillar and Base of Tongue Cancer Is Correlated to Outcome
}

\author{
CINZIA BERSANI ${ }^{1}$, LINNEA HAEGGBLOM ${ }^{1}$, RAMONA G. URSU ${ }^{1,2}$, SIMONA E. GIUSCA ${ }^{2}$, \\ LINDA MARKLUND $^{3}$, TORBJÖRN RAMQVIST ${ }^{1}$, ANDERS NÄSMAN $^{1,4^{*}}$ and TINA DALIANIS ${ }^{1 *}$ \\ ${ }^{1}$ Department of Oncology-Pathology, Karolinska Institutet, Stockholm, Sweden; \\ ${ }^{2}$ University of Medicine and Pharmacy Grigore T. Popa, Iasi, Romania; \\ ${ }^{3}$ CLINTEC, Karolinska Institutet, Stockholm, Sweden; \\ ${ }^{4}$ Department of Clinical Pathology, Karolinska University Hospital, Stockholm, Sweden
}

\begin{abstract}
Background/Aim: Human papillomavirus-positive $\left(H P V^{+}\right)$tonsillar and base of tongue squamous cell carcinoma (TSCC/BOTSCC) have better outcome than corresponding $H P V^{-}$cancers. To better individualize treatment, additional predictive markers are needed. Previously, we have shown that mutated fibroblast growth factor receptor 3 protein (FGFR3) was correlated to poorer prognosis and here FGFR3 expression was further analyzed. Patients and Methods: Onehundred-fifteen $H P V^{+} T S C C /$ BOTSCC biopsies were analyzed for FGFR3 by immunohistochemistry (IHC), and 109/115 were analyzed for FGFR3 mutations by Ion Proton sequencing, or by Competitive Allele-Specific Taqman PCR (CAST-PCR). Disease-free survival (DFS) was then calculated according to FGFR3 IHC expression. Results: CAST-PCR was useful for detecting the three most common FGFR3 mutations. Focusing especially on the 98/115 patients with HPV ${ }^{+}$TSCC/BOTSCC and wild-type FGFR3, high FGFR3 expression correlated to significantly better 3-year DFS, $p=0.043$. Conclusion: In patients with $H P V^{+} T S C C / B O T S C C$ and wild-type FGFR3, overexpression of FGFR3 was correlated with better DFS.
\end{abstract}

Patients with human papillomavirus-positive $\left(\mathrm{HPV}^{+}\right)$tonsillar and base of tongue squamous cell carcinoma (TSCC/ BOTSCC) have much better disease-free survival (DFS) than those with $\mathrm{HPV}$-negative $\left(\mathrm{HPV}^{-}\right)$cancer and most of the other head and

This article is freely accessible online.

*These Authors contributed equally to this study.

Correspondence to: Tina Dalianis, MD, Ph.D., Dept. of OncologyPathology, Karolinska Institutet, Cancer Center Karolinska, R8:01, Karolinska University Hospital, 17176 Stockholm, Sweden. Tel: +4685172764, e-mail: Tina.Dalianis@ki.se

Key Words: HPV, tonsillar cancer, base of tongue cancer, oropharyngeal cancer, FGFR3, FGFR3 immunohistochemistry, survival. neck squamous cell carcinomas (HNSCC) (1-6). Also, in many countries the incidences of TSCC/BOTSCC, the oropharyngeal squamous cell carcinoma (OPSCC) subtypes where HPV is most common, have increased (5-14). In parallel, HNSCC treatment has been intensified with hyperfractionated radiotherapy, chemoradiotherapy, targeted therapy and surgery. Most $\mathrm{HPV}^{+}$ TSCC/BOTSCC patients do not need aggressive treatment, and to reduce side-effects individualized therapy would be of benefit $(5,15,16)$. To better tailor therapy, attempts have been made to find prognostic markers (17-25). In $\mathrm{HPV}^{+}$TSCC/BOTSCC, examples of such markers are age, stage, smoking, high $\mathrm{CD}^{+}$ tumor-infiltrating lymphocyte (TIL) counts, HPV16 E2 mRNA expression, absent/low HLA class I, CD44, LMP10 expression, high expression of LRIG1 or CD98, and absence of HLA-A*02 (16-25). Mathematical models, combining some of the above markers, can identify $40-56 \%$ of patients with high probability for a 3-year DFS $(26,27)$. However, to recognize more patients that can respond to therapy and to introduce de-escalated or targeted therapy more markers are needed $(5,16)$.

To find new prognostic biomarkers, next-generation sequencing (NGS) of hot spot mutations was performed in 50 cancer related genes and found discrepancies between $\mathrm{HPV}^{+}$ and $\mathrm{HPV}^{-}$TSCC/BOTSCC (28). $\mathrm{HPV}^{+} \mathrm{TSCC}^{\mathrm{B} O T S C C}$ more frequently had PIK3CA and FGFR3 mutations, while TP53 mutations dominated in $\mathrm{HPV}^{-}$cancer, similar to data by Tinhofer et al. (29). Also $\mathrm{HPV}^{+} \mathrm{TSCC} / \mathrm{BOTSCC}$ with FGFR3 mutations was correlated with worse prognosis $(28,29)$.

Here, FGFR3 expression and mutation status were examined and correlated to outcome. FGFR3 expression was tested by immunohistochemistry (IHC). A Competitive Allele-Specific Taqman PCR (CAST-PCR) was used to detect the three most common FGFR3 mutations in samples previously not sequenced by NGS, and the two methods were compared.

\section{Patients and Methods}

Patients and tumor characteristics. Patients diagnosed from 2000 to 2011 at Karolinska University Hospital, with TSCC (ICD-10 code 
C09.0-9) or BOTSCC (ICD-10 code C01.9), treated with curative intent, were analyzed for FGFR3 by IHC (Table I). HPV ${ }^{+}$ TSCC/BOTSCC was defined as having an HPV DNA positive tumor with $16^{\mathrm{INK} 4 \mathrm{~A}}\left(\mathrm{p} 16^{+}\right)$overexpression, or expression HPV 16 E7 mRNA (30). Totally, $115 \mathrm{HPV}^{+}$TSCC/BOTSCC fulfilling these criteria, according to our previous studies $(10,14,23,28)$, were analyzed for FGFR3 expression with IHC and 98 of them had already been tested for their FGFR3 status (28).

To obtain the FGFR3 status of the remaining samples in the IHC analysis, and to compare two methods for detecting FGFR3 mutations (amplification by PCR using the Ion AMpliSeq Cancer Hotspot Panel v2 and CAST-PCR), as well as to test the validity of FGFR3 mutations as a poor prognostic marker, $228 \mathrm{HPV}^{+}$TSCC/BOTSCC samples from Karolinska University Hospital (2000-2016) were tested by CAST-PCR. Furthermore, $20 \mathrm{HPV}$ - TSCC/BOTSCC cases (from Iasu, Romania) were added for comparison.

The study was performed according to permission 2009/1278$31 / 4$ from the Ethical Committee at Karolinska Institutet and permission 3953 (2018) from the University of Medicine and Pharmacy, Grigore T Popa, Iasi, Romania.

Analysis of HPV DNA, and p16 overexpression. HPV DNA status was assayed by a PCR-based bead-based multiplex-assay on a MagPix instrument (Luminex Inc.) as described before $(23,31)$. p16 had been tested previously using the monoclonal antibody (mAb) clone JC8 (Santa Cruz Biotech, Santa Cruz, California, USA), or the E6H4 ${ }^{\mathrm{TM}}$ mouse mAb clone (CINtec ${ }^{\circledR}$, Ventana, Tucson, Arizona, USA) $(23,31)$.

Library preparation and sequencing using the Ion Ampliseq cancer hotspot panel $v 2$. Hotspot regions in 50 cancer-related genes had been tested for by us in $325 \mathrm{TSCC} / \mathrm{BOTSCC}$ by targeted PCR amplification using the Ion AMpliSeq Cancer Hotspot Panel v2 (CHPv2 - Thermo Fisher Scientific), and of these 98 were included in the FGFR IHC analysis (28).

Competitive allele-specific Taqman PCR (CAST-PCR). Detection of FGFR3 mutations was performed by Competitive Allele-Specific Taqman ${ }^{\circledR}$ PCR technology (Thermo Fischer Scientific, Waltham, MA, USA). The analysis was performed in 384-well plates, in $10 \mu \mathrm{l}$ comprising $5 \mu \mathrm{l} 2 \mathrm{X}$ Taqman Genotyping Mastermix (Thermo Fischer Scientific, Waltham, MA, USA), $0.2 \mu 1$ 50X Exogenous IPC template DNA, $1 \mu \mathrm{l}$ 10X Exogenous IPC mix, $1 \mu$ l Mutation Detection Assay, $1.8 \mu \mathrm{l}$ deionized water and $20 \mathrm{ng}$ DNA (in $1 \mu \mathrm{l}$ ). Runs were performed on an Applied Biosystems 7900HT Fast Real-Time PCR System using the following set of reaction conditions: $95^{\circ} \mathrm{C}, 10 \mathrm{~min}$ followed by 5 cycles at $92^{\circ} \mathrm{C}, 15 \mathrm{sec}$ and $58^{\circ} \mathrm{C}, 1 \mathrm{~min}$ and 40 cycles at $92^{\circ} \mathrm{C}$ for $15 \mathrm{sec}$ and $60^{\circ} \mathrm{C}$ for $1 \mathrm{~min}$. The PCR result was analyzed with the SDS 2.3 software program and Mutation Detector Software 2.0 (Thermo Fischer Scientific, Waltham, MA, USA). Ct was determined for exogenous IPC (Internal Passive Control) reagents added to each reaction to evaluate PCR failure or inhibition in a reaction. The Mutation Detection Assays were Hs00000811_mu, Hs00000812_mu, Hs00001342_mu, which detects variants p.R248C, p.S249C and p.K650Q in FGFR3 gene respectively, and reference assay Hs00001015_rf was used for detection of wild-type FGFR3.

Immunohistochemistry for FGFR3. Evaluation of FGFR3 protein expression by IHC was done on $4 \mu \mathrm{m}$ FFPE sections with the mouse mAb FGFR3 clone B-9 (Santa Cruz Biotechnology, Heidelberg, Germany), using an avidin-biotin peroxidase method as previously described $(21,23)$. Staining was blindly evaluated by two researchers, including one pathologist. Tumor staining intensity was scored as 0 , absent; 1 , weak; 2, moderate; and 3, strong, and tumor percentage of staining was assessed to the nearest $10 \%$. For cases where the evaluation differed, a consensus was reached. Cases where the staining was not possible to evaluate were excluded.

Statistical analysis. Categorial variables (FGFR3 IHC and FGFR3 mutation data) were compared with the Chi2-test. Clinical outcome was measured as 3-year DFS or 3-year overall survival (OS). DFS was defined as time from diagnosis until date of relapse in disease and OS defined as time from diagnosis until date of death. Patients never tumor-free were censored on day 0 , while patients dying without prior recurrence were censored at the time-point, when assessing DFS. Survival curves with 3-year DFS and OS were calculated using the Kaplan-Meier method and differences in survival were tested using the log-rank test. All statistical tests were performed in STATA (STATA v11, StataCorp, 4905 Lakeway Dr, College Station, TX 77845, USA).

\section{Results}

Analysis of FGFR3 mutations in HPV ${ }^{+}$TSCC/BOTSCC by CAST-PCR. Totally $115 \mathrm{HPV}^{+}$TSCC/BOTSCC samples were evaluated for FGFR3 expression by IHC and the characteristics of the patients and their tumors are depicted in Table I. Of the $\mathrm{HPV}^{+}$TSCC/BOTSCC, 98 had been analyzed for mutations by targeted amplification using the Ion AMpliSeq Cancer Hotspot Panel v2, and of these 10 had mutations within the FGFR3 region (28). Of the remaining 17 samples, 1/11 successfully analyzed for FGFR3 mutations by CAST-PCR had an FGFR3 mutation, while for six samples FGFR 3 status could not be determined due to lack of material. Thus, by sequencing or CAST-PCR, 11/109 $\mathrm{HPV}^{+}$TSCC/BOTSCC exhibited FGFR 3 mutations, while 98/109 samples were regarded as having wild-type FGFR3.

To analyze the prognostic importance of FGFR3 mutations in a larger set, and since CAST-PCR for analysis of FGFR3 has, to our knowledge, not been compared to sequencing with the Ion AMpliSeq Cancer Hotspot Panel v2, 228 new $\mathrm{HPV}^{+}$ TSCC/BOTSCC samples (not analyzed for FGFR3 mutations before (28)) were tested by CAST-PCR. Totally 196/228 $(86 \%)$ samples passed the quality requirements, and of these $16 / 196$ (8.6\%) exhibited FGFR3 mutations, similar to the $5.7 \%$ obtained by sequencing (28), for details see Table II. The COSM715-p.S249C mutation dominated with a frequency of $5 \%$ in the CAST-PCR analyzed cohort as compared to (4.3\%) previously obtained by sequencing (28) (Table II).

Furthermore, when $15 \mathrm{HPV}^{+}$TSCC/BOTSCC samples, determined as having FGFR 3 mutations when tested by NGS sequencing (28), were analyzed also by CAST-PCR, 13/15, (87\%) of these FGFR3 mutations were confirmed. In addition, 16/16 (100\%) of the previously NGS sequenced $\mathrm{HPV}^{+}$TSCC/BOTSCC samples, determined as having wildtype $F G F R 3$, were also classified as having wild-type FGFR3 when analyzed by CAST-PCR (data not shown). 
Table I. Patient and tonsillar and base of tongue squamous cell carcinoma characteristics.

\begin{tabular}{|c|c|c|c|c|c|c|c|c|c|}
\hline \multicolumn{2}{|c|}{ Patient and tumor characteristics } & \multicolumn{2}{|c|}{$\begin{array}{l}\text { Absent/low FGFR3 } \\
\text { expression } \\
(\mathrm{N}=28)\end{array}$} & \multicolumn{2}{|c|}{$\begin{array}{c}\text { Medium FGFR3 } \\
\text { expression } \\
(\mathrm{N}=39)\end{array}$} & \multicolumn{2}{|c|}{$\begin{array}{l}\text { High FGFR3 } \\
\text { expression } \\
(\mathrm{N}=48)\end{array}$} & \multicolumn{2}{|c|}{$\begin{array}{l}\text { All patients with } \\
\text { FGFR IHC staining } \\
\qquad(\mathrm{N}=115)\end{array}$} \\
\hline & & $\mathrm{N}$ & $\%$ & $\mathrm{~N}$ & $\%$ & $\mathrm{~N}$ & $\%$ & $\mathrm{~N}$ & $\%$ \\
\hline Age & Mean (years) & \multicolumn{2}{|c|}{60} & \multicolumn{2}{|c|}{61} & \multicolumn{2}{|c|}{58} & \multicolumn{2}{|c|}{59.6} \\
\hline Median (years) & & \multicolumn{2}{|c|}{60} & \multicolumn{2}{|c|}{60} & \multicolumn{2}{|c|}{59} & \multicolumn{2}{|c|}{59.5} \\
\hline Range (years) & & \multicolumn{2}{|c|}{$42-78$} & \multicolumn{2}{|c|}{$30-90$} & \multicolumn{2}{|c|}{$39-78$} & \multicolumn{2}{|c|}{$30-90$} \\
\hline \multirow[t]{4}{*}{ Diagnosis } & Base of tongue cancer & & & & & & & & \\
\hline & ICD-10: C01.9 & 7 & $25 \%$ & 11 & $28 \%$ & 13 & $27 \%$ & 31 & $27 \%$ \\
\hline & Tonsillar cancer & & & & & & & & \\
\hline & ICD-10: C09.0, C09.1, C09.8, C09.9 & 21 & $75 \%$ & 28 & $72 \%$ & 35 & $73 \%$ & 84 & $73 \%$ \\
\hline \multirow[t]{2}{*}{ Gender } & Male & 22 & $80 \%$ & 28 & $72 \%$ & 34 & $71 \%$ & 83 & $72 \%$ \\
\hline & Female & 6 & $20 \%$ & 11 & $28 \%$ & 14 & $29 \%$ & 32 & $28 \%$ \\
\hline \multirow[t]{3}{*}{ Tumor differentiation } & Poor & 19 & $64 \%$ & 28 & $72 \%$ & 33 & $69 \%$ & 80 & $70 \%$ \\
\hline & Moderate & 9 & $36 \%$ & 11 & $28 \%$ & 12 & $25 \%$ & 32 & $28 \%$ \\
\hline & Well & 0 & $0 \%$ & 0 & $0 \%$ & 3 & $6 \%$ & 3 & $3 \%$ \\
\hline \multirow[t]{4}{*}{ Tumor size } & $\mathrm{T} 1$ & 4 & $14 \%$ & 7 & $18 \%$ & 18 & $38 \%$ & 29 & $25 \%$ \\
\hline & $\mathrm{T} 2$ & 11 & $39 \%$ & 16 & $41 \%$ & 18 & $38 \%$ & 45 & $39 \%$ \\
\hline & $\mathrm{T} 3$ & 8 & $29 \%$ & 8 & $21 \%$ & 1 & $2 \%$ & 17 & $15 \%$ \\
\hline & $\mathrm{T} 4$ & 5 & $18 \%$ & 8 & $21 \%$ & 11 & $23 \%$ & 24 & $21 \%$ \\
\hline \multirow[t]{7}{*}{ Nodal disease } & No & 3 & $11 \%$ & 7 & $18 \%$ & 2 & $4 \%$ & 12 & $10 \%$ \\
\hline & $\mathrm{N} 1$ & 4 & $14 \%$ & 5 & $13 \%$ & 16 & $33 \%$ & 26 & $23 \%$ \\
\hline & $\mathrm{N} 2 \mathrm{a}$ & 5 & $18 \%$ & 5 & $13 \%$ & 4 & $8 \%$ & 14 & $12 \%$ \\
\hline & $\mathrm{N} 2 \mathrm{~b}$ & 13 & $64 \%$ & 12 & $31 \%$ & 21 & $44 \%$ & 46 & $40 \%$ \\
\hline & $\mathrm{N} 2 \mathrm{c}$ & 3 & $11 \%$ & 5 & $13 \%$ & 4 & $8 \%$ & 12 & $10 \%$ \\
\hline & $\mathrm{N} 3$ & 0 & $0 \%$ & 2 & $5 \%$ & 1 & $2 \%$ & 3 & $3 \%$ \\
\hline & $\mathrm{NX}$ & 0 & $0 \%$ & 2 & $5 \%$ & 0 & $0 \%$ & 2 & $2 \%$ \\
\hline \multirow[t]{3}{*}{ Distant metastasis } & M0 & 26 & $93 \%$ & 39 & $100 \%$ & 47 & $98 \%$ & 112 & $97 \%$ \\
\hline & M1 & 2 & $7 \%$ & 0 & $0 \%$ & 0 & $0 \%$ & 2 & $2 \%$ \\
\hline & MX & 0 & $0 \%$ & 0 & $0 \%$ & 1 & $2 \%$ & 1 & $1 \%$ \\
\hline Tumor stage & I & 4 & $14 \%$ & 4 & $10 \%$ & 18 & $38 \%$ & 26 & $23 \%$ \\
\hline (AJCC 8th Edition) & II & 17 & $61 \%$ & 22 & $56 \%$ & 19 & $40 \%$ & 58 & $50 \%$ \\
\hline & III & 5 & $18 \%$ & 10 & $26 \%$ & 10 & $21 \%$ & 25 & $22 \%$ \\
\hline & IV & 2 & $7 \%$ & 1 & $3 \%$ & 0 & $0 \%$ & 3 & $2.5 \%$ \\
\hline & Unknown & 0 & $0 \%$ & 2 & $5 \%$ & 1 & $2 \%$ & 3 & $2.5 \%$ \\
\hline Treatment & Induction chemotherapy & & & & & & & & \\
\hline & and radiation (conventional/accelerated) & 9 & $32 \%$ & 13 & $33 \%$ & 20 & $42 \%$ & 42 & $37 \%$ \\
\hline & Radiation only (conventional/accelerated) & 19 & $68 \%$ & 26 & $67 \%$ & 28 & $58 \%$ & 73 & $63 \%$ \\
\hline Smoking & Never & 8 & $29 \%$ & 18 & $46 \%$ & 16 & $33 \%$ & 42 & $37 \%$ \\
\hline & Former ( $>15$ years ago) & 4 & $14 \%$ & 6 & $15 \%$ & 6 & $13 \%$ & 16 & $14 \%$ \\
\hline & Former $(<15$ years ago $)$ & 4 & $14 \%$ & 8 & $21 \%$ & 7 & $15 \%$ & 19 & $17 \%$ \\
\hline & Current upon diagnosis & 12 & $43 \%$ & 7 & $18 \%$ & 19 & $40 \%$ & 38 & $33 \%$ \\
\hline FGFR3 status & Wild type & 22 & $78 \%$ & 37 & $95 \%$ & 39 & $81 \%$ & 98 & $85 \%$ \\
\hline & Mutated & 3 & $11 \%$ & 1 & $3 \%$ & 7 & $15 \%$ & 11 & $10 \%$ \\
\hline & Not tested & 3 & $11 \%$ & 1 & $3 \%$ & 2 & $4 \%$ & 6 & $5 \%$ \\
\hline
\end{tabular}

Finally, to confirm that FGFR3 mutations were less common in $\mathrm{HPV}^{-}$TSCC/BOTSCC, CAST-PCR was performed also in $20 \mathrm{HPV}^{-}$TSCC/BOTSCC from Iasi, Romania and all these exhibited wild-type FGFR3. This finding was in concordance with the previous sequencing data showing a very low frequency of mutated FGFR3 (1/46, $2.1 \%$ ) among $\mathrm{HPV}^{-}$samples (28).
FGFR3 expression in HPV ${ }^{+}$TSCC/BOTSCC analyzed by immunohistochemistry. FGFR3 staining was evaluated as a percentage of tumor cells per intensity group (defined as absent/low, medium or strong expression) (Table I and Figure 1). Tumor FGFR3 staining was heterogeneous and different staining intensities could sometimes be observed in the same tumor. Tumors were therefore grouped together by 
Table II. Samples tested for presence of FGFR3 mutations by CAST-PCR compared to prevalence obtained using sequencing with the Ion AMpliSeq Cancer Hotspot Panel v2.

\begin{tabular}{lccc}
\hline Samples and mutants & No. of samples & Percentage by CAST-PCR & $\begin{array}{c}\text { Percentage by the Ion AmpliSeq } \\
\text { Cancer Hostpot Panel v2 ref }\end{array}$ \\
\hline Samples tested & 228 & $14 \%$ & 297 \\
Non passed & 32 & $86 \%$ & $6 \%$ \\
Passed & 196 & $3 \%$ & $1 \%$ \\
COSM714 - p.R248C & 6 & $5 \%$ & $4,3 \%$ \\
COSM715 - p.S249C & 9 & $0,6 \%$ & $0,35 \%$ \\
COSM726 - p.K650Q & 1 & $8 \%$ & $6 \%$ \\
\hline Total & $16 / 196$ & $8 \%$ \\
\hline
\end{tabular}

their highest intensity staining to create three intensity groups (absent/low, medium and strong). In total, 48 samples were defined as having high, 39 samples having medium, and 28 samples having absent/low FGFR3 expression. Among those with wild-type FGFR3, 39 had high, 37 had medium, and 22 had absent/low expression. Among those with mutated FGFR3, seven samples had high, one medium, and three had absent/low FGFR3 expression. There were no major differences in high FGFR3 expression between samples with wild-type or mutated FGFR3 $(p=0.2)$.

FGFR3 expression in relation to clinical outcome in $\mathrm{HPV}^{+}$ TSCC/BOTSCC. FGFR3 expression defined by IHC was correlated to outcome defined as 3-year DFS or OS. Initially, attempts were made to evaluate survival for all 115 patients separated into three groups with low, medium and high FGR3 expression in the tumors. This analysis indicated a similar survival for patients with low and medium FGFR3 expression, and therefore the latter two were grouped together. Furthermore, it seemed reasonable to analyze survival only among the 98 patients with $\mathrm{HPV}^{+}$ TSCC/BOTSCC and wild-type FGFR3 and exclude the 11 cases with FGFR3 mutations and the six patients with unknown FGFR3 mutation status.

Upon this stratification (39 patients with high and 59 with medium/low FGFR3 expression) a clear picture emerged disclosing a significant correlation between high FGFR3 expression compared to having medium/low FGFR3 expression and better DFS (log-rank test, $p=0.043$ ) (Figure 2). There was, however, no significant difference between the groups with regard to OS (data not shown). A similar correlation was attempted for the 11 mutated FGFR3 cases, but no specific differences were revealed (log-rank test, $p=0.5$ ).

FGFR3 mutation status in relation to clinical outcome. Clinical outcome was also examined for the 109 patients with $\mathrm{HPV}^{+}$TSCC/BOTSCC, with known FGFR3 wild-type or mutant status. Notably, however, 98 of these 109 patients with $\mathrm{HPV}^{+}$TSCC/BOTSCC had already been included in a survival analysis, in our previous report, showing that having mutated FGFR3 was correlated to worse DFS (28). After analyzing the clinical outcome for all 109 patients with $\mathrm{HPV}^{+}$TSCC/BOTSCC in this study, it was still clear that patients having wild-type FGFR3 in their tumors had better DFS than those with FGFR3 mutations in their tumors (logrank test, $p=0.007)$. An analogous survival analysis was performed for 121 patients having completed a 3-year follow up period among the 196 patients with adequate data obtained in the new CAST-PCR tested cohort. However, no significant correlation between FGFR3 mutation status and survival could be observed.

\section{Discussion}

Here FGFR3 expression was analyzed by IHC in a cohort of $115 \mathrm{HPV}^{+}$TSCC/BOTSCC and correlated to outcome in patients with tumors with wild-type FGFR3. High FGFR3 expression was found in $40 \%$ of the tumors, and for those with wild-type FGFR3, high expression correlated to better DFS as compared to those with medium/low FGFR3 expression. A similar analysis was not possible to perform in the FGFR3 mutated group, due to few patients.

There is limited information on FGFR3 expression in OPSCC/TSCC/BOTSCC. One report by Koole et al. (32) analyzed overexpression of FGFR3, (using the same FGFR3 antibody as in this study) in oral squamous cell carcinoma (OSCC) and OPSCC and found that FGFR3 was overexpressed in $48 \%$ and $59 \%$ of OSCC and OPSCC respectively, which is similar to our data. Nevertheless, FGFR3 expression was not correlated to DFS or OS irrespective of whether OPSCC was separated into $\mathrm{HPV}^{+}$ and $\mathrm{HPV}^{-}$cases (32). Consequently, our two studies differ. However, in that study there were only $18 \mathrm{HPV}^{+}$OPSCC cases, and the analysis did not take into consideration whether FGFR3 was wild-type or mutated (32). Nonetheless, the authors concluded, similar to us in our sequencing report, 

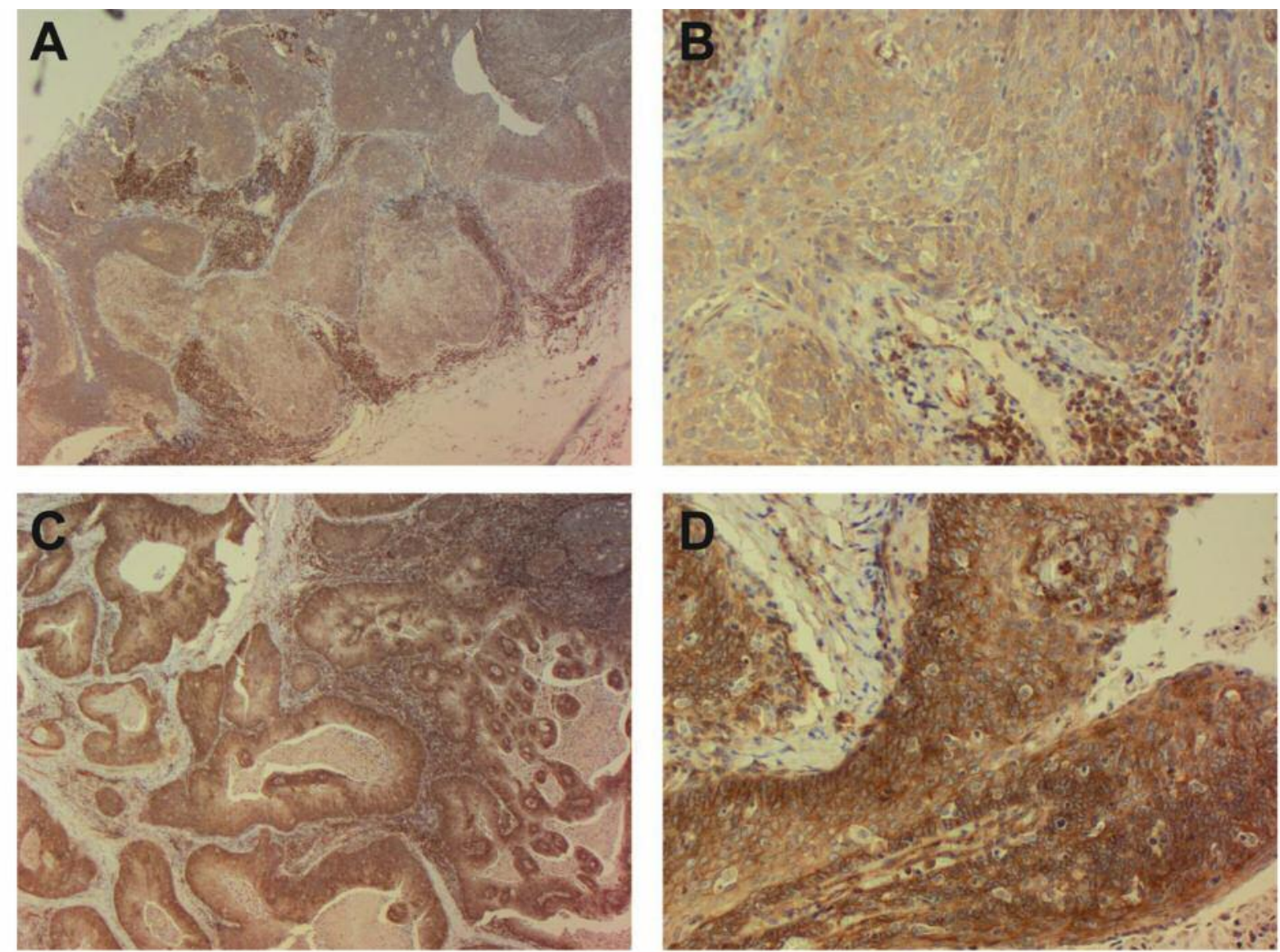

Figure 1. Immunohistochemistry of FGFR3. A and B: High FGFR3 expression in HPV ${ }^{+}$TSCC, $4 \times$ and 20x respectively; C and D: Low FGFR3 expression in $\mathrm{HPV}^{+} \mathrm{TSCC}, 4 \times$ and $20 \times$ respectively.

that FGFR3 could serve as a therapeutic target for FGFR3directed therapies $(28,32)$.

FGFR3 expression has been investigated in more detail in other tumors, especially in e.g. bladder cancer (33-35). Bertz et al. (33) examined the role of angiogenesis and FGFR3 protein expression in bladder cancer and showed that high FGFR3 expression was correlated to better survival in a multivariate analysis. The authors suggested that FGFR3 could be a potential therapeutic target also from the angiogenesis perspective (33).

FGFR3 has been studied in brain tumors, and high expression of FGFR3 has been shown to be common in aggressive ependymomas, although it was not likely driven by genetic changes (36). In gliomas strong FGFR3 expression has been detected upon FGFR3 fusions and in another report FGFR3 expression was mainly correlated to the presence of squamous cell carcinoma, but FGFR3 expression was not correlated to survival in any of these tumors (37).

Here, using CAST-PCR, the presence of the three most common FGFR3 mutations previously observed by sequencing using the Ion AMpliSeq Cancer Hotspot Panel v2 in a new cohort, including also some tumors tested for FGFR3 IHC was evaluated (28). In this new cohort we could show that $8.6 \%$ of the tumors exhibited FGFR3 mutations. This was insignificantly higher than that in our previous study $(5.4 \%)$ and similar to that (10\%) recently published by Mirghani et al. $(28,38)$. The method discovered $87 \%$ of the mutations detected before using the Ion AMpliSeq Cancer Hotspot Panel v2 and did not reveal any additional FGFR3 mutations in "negative control samples", so the compatibility was relatively good between the two methods. Presently however, we do not have an explanation for the discrepancy between these methods with regard to mutations in some tumors.

There are some limitations in this study. First of all, here only three of the most common FGFR3 mutations were tested and although other FGFR3 mutations are rare, they may still be present in the analyzed material. Furthermore, we do not know if some tumors have other genetic changes in FGFR3 e.g. amplifications or translocations. In addition, FGFR3 mutations are not very frequent, and since $\mathrm{HPV}^{+}$TSCC/BOTSCC in 


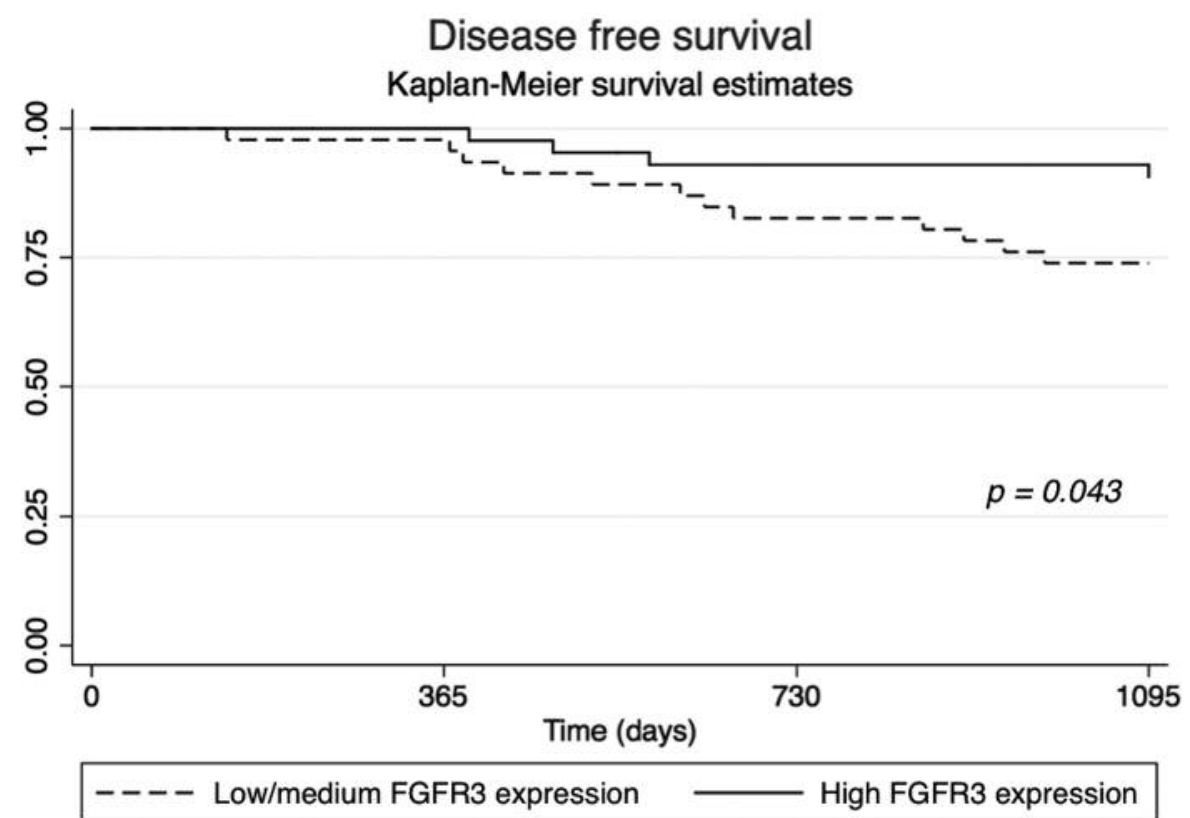

Figure 2. Disease-free survival (DSF) for patients with $\mathrm{HPV}^{+}$TSCC/BOTSCC stratified for low/medium and high FGFR3 expression. Cumulative DSF for HPV $V^{+}$TSCC/BOTSCC stratified for medium/low FGFR3 and high expression. Differences in survival were analyzed with the log-rank test.

general have a very favorable outcome, analysis of $\mathrm{HPV}^{+}$ TSCC/BOTSCC from a large number of patients is required to fully evaluate survival in relation to FGFR3 mutation status. Therefore, with a larger cohort and a longer follow up time it is possible to obtain different results. Nonetheless, the finding that high FGFR3 expression among patients with wild-type FGFR3 was correlated to better DFS may be of clinical value.

FGFR3 has, as indicated above, been suggested to be useful for targeted therapy. However, given the fact that overexpression of wild-type FGFR3 is a prognostic favorable factor, caution is required, and further studies are needed before initiating clinical studies on $\mathrm{HPV}^{+}$TSCC/BOTSCC.

To conclude, in patients with $\mathrm{HPV}^{+} \mathrm{TSCC} / \mathrm{BOTSCC}$ and wild-type FGFR3, overexpression of FGFR3 by IHC was correlated to better DFS. CAST-PCR was efficient in detecting the majority of FGFR 3 mutations also detected by Ion AMpliSeq Cancer Hotspot Panel v2, but upon validation of the role of FGFR3 mutations as a prognostic marker, our previous findings were not possible to confirm.

\section{Acknowledgements}

This research was supported by the Swedish Cancer Foundation, the Stockholm Cancer Society, the Swedish Cancer and Allergy Foundation, the Sigurd and Elsa Goljes Foundation, Swedish Society for Medical Research (SSMF), the Royal Swedish Academy of Sciences, the Stockholm City Council and Karolinska Institutet, Sweden. RGU was funded by the University of Medicine and Pharmacy, Grigore T Popa, Iasi, based on contract no. 30336/28 12-2017.

\section{References}

1 Mellin H, Friesland S, Lewensohn R, Dalianis T and MunckWikland E: Human papillomavirus (hpv) DNA in tonsillar cancer: Clinical correlates, risk of relapse, and survival. Int J Cancer 89(3): 300-304, 2000.

2 Dahlgren L, Dahlstrand HM, Lindquist D, Hogmo A, Bjornestal L, Lindholm J, Lundberg B, Dalianis T and Munck-Wikland E: Human papillomavirus is more common in base of tongue than in mobile tongue cancer and is a favorable prognostic factor in base of tongue cancer patients. Int J Cancer 112(6): 1015-1019, 2004.

3 Attner P, Du J, Nasman A, Hammarstedt L, Ramqvist T, Lindholm J, Marklund L, Dalianis T and Munck-Wikland E: Human papillomavirus and survival in patients with base of tongue cancer. Int J Cancer 128(12): 2892-2897, 2011.

4 Sivars L, Nasman A, Tertipis N, Vlastos A, Ramqvist T, Dalianis T, Munck-Wikland E and Nordemar S: Human papillomavirus and p53 expression in cancer of unknown primary in the head and neck region in relation to clinical outcome. Cancer Med 3(2): 376-384, 2014.

5 Dalianis T: Human papillomavirus and oropharyngeal cancer, the epidemics, and significance of additional clinical biomarkers for prediction of response to therapy (review). Int J Oncol 44(6): 1799-1805, 2014.

6 Marklund L, Nasman A, Ramqvist T, Dalianis T, MunckWikland E and Hammarstedt L: Prevalence of human papillomavirus and survival in oropharyngeal cancer other than tonsil or base of tongue cancer. Cancer Med 1(1): 82-88, 2012.

7 Robinson KL and Macfarlane GJ: Oropharyngeal cancer incidence and mortality in scotland: Are rates still increasing? Oral Oncol 39(1): 31-36, 2003. 
8 Hammarstedt L, Lindquist D, Dahlstrand H, Romanitan M, Dahlgren LO, Joneberg J, Creson N, Lindholm J, Ye W, Dalianis $\mathrm{T}$ and Munck-Wikland E: Human papillomavirus as a risk factor for the increase in incidence of tonsillar cancer. Int $\mathrm{J}$ Cancer 119(11): 2620-2623, 2006.

9 Sturgis EM and Cinciripini PM: Trends in head and neck cancer incidence in relation to smoking prevalence: An emerging epidemic of human papillomavirus-associated cancers? Cancer 110(7): 1429-1435, 2007.

10 Nasman A, Attner P, Hammarstedt L, Du J, Eriksson M, Giraud G, Ahrlund-Richter S, Marklund L, Romanitan M, Lindquist D, Ramqvist T, Lindholm J, Sparen P, Ye W, Dahlstrand H, MunckWikland $\mathrm{E}$ and Dalianis T: Incidence of human papillomavirus (hpv) positive tonsillar carcinoma in stockholm, sweden: An epidemic of viral-induced carcinoma? Int J Cancer 125(2): 362366, 2009.

11 Braakhuis BJ, Visser O and Leemans CR: Oral and oropharyngeal cancer in the netherlands between 1989 and 2006: Increasing incidence, but not in young adults. Oral Oncol 45(9): e85-89, 2009.

12 Attner P, Du J, Nasman A, Hammarstedt L, Ramqvist T, Lindholm J, Marklund L, Dalianis T and Munck-Wikland E: The role of human papillomavirus in the increased incidence of base of tongue cancer. Int J Cancer 126(12): 2879-2884, 2010.

13 Chaturvedi AK, Engels EA, Pfeiffer RM, Hernandez BY, Xiao W, Kim E, Jiang B, Goodman MT, Sibug-Saber M, Cozen W, Liu L, Lynch CF, Wentzensen N, Jordan RC, Altekruse S, Anderson WF, Rosenberg PS and Gillison ML: Human papillomavirus and rising oropharyngeal cancer incidence in the united states. J Clin Oncol 29(32): 4294-4301, 2011.

14 Nasman A, Nordfors C, Holzhauser S, Vlastos A, Tertipis N, Hammar U, Hammarstedt-Nordenvall L, Marklund L, MunckWikland E, Ramqvist T, Bottai M and Dalianis T: Incidence of human papillomavirus positive tonsillar and base of tongue carcinoma: A stabilisation of an epidemic of viral induced carcinoma? Eur J Cancer 51(1): 55-61, 2015.

15 Mirghani H, Amen F, Blanchard P, Moreau F, Guigay J, Hartl DM and Lacau St Guily J: Treatment de-escalation in hpvpositive oropharyngeal carcinoma: Ongoing trials, critical issues and perspectives. Int J Cancer 136(7): 1494-1503, 2015.

16 Nasman A, Bersani C, Lindquist D, Du J, Ramqvist T and Dalianis T: Human papillomavirus and potentially relevant biomarkers in tonsillar and base of tongue squamous cell carcinoma. Anticancer Res 37(10): 5319-5328, 2017.

17 Ang KK, Harris J, Wheeler R, Weber R, Rosenthal DI, NguyenTan PF, Westra WH, Chung CH, Jordan RC, Lu C, Kim H, Axelrod R, Silverman CC, Redmond KP and Gillison ML: Human papillomavirus and survival of patients with oropharyngeal cancer. N Engl J Med 363(1): 24-35, 2010.

18 Nasman A, Andersson E, Marklund L, Tertipis N, HammarstedtNordenvall L, Attner P, Nyberg T, Masucci GV, Munck-Wikland E, Ramqvist T and Dalianis T: Hla class $\mathrm{i}$ and ii expression in oropharyngeal squamous cell carcinoma in relation to tumor hpv status and clinical outcome. PLoS One 8(10): e77025, 2013.

19 Nasman A, Nordfors C, Grun N, Munck-Wikland E, Ramqvist T, Marklund L, Lindquist D and Dalianis T: Absent/weak cd44 intensity and positive human papillomavirus (hpv) status in oropharyngeal squamous cell carcinoma indicates a very high survival. Cancer Med 2(4): 507-518, 2013.

20 Tertipis N, Haeggblom L, Nordfors C, Grun N, Nasman A, Vlastos A, Dalianis T and Ramqvist T: Correlation of $1 \mathrm{mp} 10$ expression and clinical outcome in human papillomavirus (hpv) positive and hpv-negative tonsillar and base of tongue cancer. PLoS One 9(4): e95624, 2014.

21 Lindquist D, Nasman A, Tarjan M, Henriksson R, Tot T, Dalianis $\mathrm{T}$ and Hedman H: Expression of lrig1 is associated with good prognosis and human papillomavirus status in oropharyngeal cancer. Br J Cancer 110(7): 1793-1800, 2014.

22 Tertipis N, Villabona L, Nordfors C, Nasman A, Ramqvist T, Vlastos A, Masucci G and Dalianis T: Hla-a*02 in relation to outcome in human papillomavirus positive tonsillar and base of tongue cancer. Anticancer Res 34(5): 2369-2375, 2014.

23 Nordfors C, Grun N, Tertipis N, Ahrlund-Richter A, Haeggblom L, Sivars L, Du J, Nyberg T, Marklund L, Munck-Wikland E, Nasman A, Ramqvist T and Dalianis T: $\mathrm{Cd}^{+}$and $\mathrm{cd} 4^{+}$tumour infiltrating lymphocytes in relation to human papillomavirus status and clinical outcome in tonsillar and base of tongue squamous cell carcinoma. Eur J Cancer 49(11): 2522-2530, 2013.

24 Ramqvist T, Mints M, Tertipis N, Nasman A, Romanitan M and Dalianis T: Studies on human papillomavirus (hpv) 16 e2, e5 and e7 mrna in hpv-positive tonsillar and base of tongue cancer in relation to clinical outcome and immunological parameters. Oral Oncol 51(12): 1126-1131, 2015.

25 Rietbergen MM, Martens-de Kemp SR, Bloemena E, Witte BI, Brink A, Baatenburg de Jong RJ, Leemans CR, Braakhuis BJ and Brakenhoff RH: Cancer stem cell enrichment marker cd98: A prognostic factor for survival in patients with human papillomavirus-positive oropharyngeal cancer. Eur J Cancer 50(4): 765-773, 2014.

26 Tertipis N, Hammar U, Nasman A, Vlastos A, Nordfors C, Grun N, Ahrlund-Richter A, Sivars L, Haeggblom L, Marklund L, Hammarstedt-Nordenvall L, Chaturvedi AK, Munck-Wikland E, Ramqvist T, Bottai M and Dalianis T: A model for predicting clinical outcome in patients with human papillomavirus-positive tonsillar and base of tongue cancer. Eur J Cancer 51(12): 15801587, 2015.

27 Bersani C, Mints M, Tertipis N, Haeggblom L, Sivars L, Ahrlund-Richter A, Vlastos A, Smedberg C, Grun N, MunckWikland E, Nasman A, Ramqvist T and Dalianis T: A model using concomitant markers for predicting outcome in human papillomavirus positive oropharyngeal cancer. Oral Oncol 68 : 53-59, 2017.

28 Bersani C, Sivars L, Haeggblom L, DiLorenzo S, Mints M, Ahrlund-Richter A, Tertipis N, Munck-Wikland E, Nasman A, Ramqvist $\mathrm{T}$ and Dalianis $\mathrm{T}$ : Targeted sequencing of tonsillar and base of tongue cancer and human papillomavirus positive unknown primary of the head and neck reveals prognostic effects of mutated fgfr3. Oncotarget 8(21): 35339-35350, 2017.

29 Tinhofer I, Budach V, Saki M, Konschak R, Niehr F, Johrens K, Weichert W, Linge A, Lohaus F, Krause M, Neumann K, Endris V, Sak A, Stuschke M, Balermpas P, Rodel C, Avlar M, Grosu AL, Abdollahi A, Debus J, Belka C, Pigorsch S, Combs SE, Monnich D, Zips D, Baumann M and Dktk ROG: Targeted next-generation sequencing of locally advanced squamous cell carcinomas of the head and neck reveals druggable targets for improving adjuvant chemoradiation. Eur J Cancer 57: 78-86, 2016.

30 Smeets SJ, Hesselink AT, Speel EJ, Haesevoets A, Snijders PJ, Pawlita M, Meijer CJ, Braakhuis BJ, Leemans CR and Brakenhoff RH: A novel algorithm for reliable detection of human papillomavirus in paraffin embedded head and neck cancer specimen. Int J Cancer 121(11): 2465-2472, 2007. 
31 Dalianis T, Grun N, Koch J, Vlastos A, Tertipis N, Nordfors C, Nasman A, Wendt M, Romanitan M, Bersani C, Munck-Wikland E and Ramqvist T: Human papillomavirus DNA and p16(ink4a) expression in hypopharyngeal cancer and in relation to clinical outcome, in stockholm, sweden. Oral Oncol 51(9): 857-861, 2015.

32 Koole K, van Kempen PM, Swartz JE, Peeters T, van Diest PJ, Koole R, van Es RJ and Willems SM: Fibroblast growth factor receptor 3 protein is overexpressed in oral and oropharyngeal squamous cell carcinoma. Cancer Med 5(2): 275-284, 2016.

33 Bertz S, Abee C, Schwarz-Furlan S, Alfer J, Hofstadter F, Stoehr $\mathrm{R}$, Hartmann A and Gaumann AK: Increased angiogenesis and fgfr protein expression indicate a favourable prognosis in bladder cancer. Virchows Arch 465(6): 687-695, 2014.

34 Tomlinson DC, Baldo O, Harnden P and Knowles MA: Fgfr3 protein expression and its relationship to mutation status and prognostic variables in bladder cancer. J Pathol 213(1): 91-98, 2007.

35 Gust KM, McConkey DJ, Awrey S, Hegarty PK, Qing J, Bondaruk J, Ashkenazi A, Czerniak B, Dinney CP and Black PC: Fibroblast growth factor receptor 3 is a rational therapeutic target in bladder cancer. Mol Cancer Ther 12(7): 1245-1254, 2013.
36 Lehtinen B, Raita A, Kesseli J, Annala M, Nordfors K, Yli-Harja O, Zhang W, Visakorpi T, Nykter M, Haapasalo H and Granberg $\mathrm{KJ}$ : Clinical association analysis of ependymomas and pilocytic astrocytomas reveals elevated fgfr 3 and fgfr 1 expression in aggressive ependymomas. BMC Cancer 17(1): 310, 2017.

37 Granberg KJ, Annala M, Lehtinen B, Kesseli J, Haapasalo J, Ruusuvuori P, Yli-Harja O, Visakorpi T, Haapasalo H, Nykter M and Zhang W: Strong fgfr3 staining is a marker for fgfr3 fusions in diffuse gliomas. Neuro Oncol 19(9): 1206-1216, 2017.

38 Mirghani H, Lacroix L, Rossoni C, Sun R, Auperin A, Casiraghi O, Villepelet A, Lacave R, Faucher G, Marty V, Ferte C, Soria JC and Even C: Does smoking alter the mutation profile of human papillomavirus-driven head and neck cancers? Eur J Cancer 94: 61-69, 2018.
Received June 13, 2018

Revised June 25, 2018

Accepted June 26, 2018 